\title{
Questes
}

vestes Revue pluridisciplinaire d'études médiévales

\section{Les manifestations divines : introduction}

\section{Céline Ménager et Isabelle Coumert}

\section{(2) OpenEdition}

Journals

Édition électronique

URL : http://journals.openedition.org/questes/1001

DOI : 10.4000/questes.1001

ISSN : 2109-9472

\section{Éditeur}

Les Amis de Questes

\section{Édition imprimée}

Date de publication : 15 septembre 2010

Pagination : 5-22

ISSN : 2102-7188

\section{Référence électronique}

Céline Ménager et Isabelle Coumert, «Les manifestations divines : introduction », Questes [En ligne],

19 | 2010, mis en ligne le 01 janvier 2014, consulté le 23 septembre 2020. URL : http:// journals.openedition.org/questes/1001 ; DOI : https://doi.org/10.4000/questes.1001 


\section{Les manifestations divines Introduction}

\section{Céline MenAGER et Isabelle COUMERT}

Si l'expression de «manifestation divine» ne semble pas ambiguë de prime abord, elle reste délicate à définir. Une recherche d'ouvrages portant sur les manifestations divines dans les catalogues de bibliothèques en utilisant cette expression risque de vite tourner court; il en sera de même si l'on consulte les divers dictionnaires portant sur la religion chrétienne. C'est que le pluriel de cette expression n'est pas innocent et annonce assez bien le problème que pose la définition des manifestations divines: ces dernières sont des phénomènes très divers et nous ne disposons pas d'un terme unique susceptible de regrouper des formes aussi variées.

La manifestation divine a donné lieu au Moyen Âge à de nombreuses créations, tant littéraires qu'artistiques, et a fourni un important fonds iconographique. Mais au cœur d'une période où foisonnent et coexistent phénomènes merveilleux, créatures fantastiques, fées et magiciens, la manifestation divine n'est qu'une des nombreuses formes d'un surnaturel apparemment omniprésent, d'où une certaine difficulté à distinguer les manifestations divines du reste du surnaturel.

\section{Une expression difficile à définir}

Le latin du Moyen Âge utilisait différents vocables pour évoquer les phénomènes merveilleux ou surnaturels - mirabilia, miraculum, prodigium, signum, virtus... - sans qu'il nous soit toujours possible de définir très précisément ces termes. Reprenant Gervais de Tilbury, Jacques 
Le Goff $^{1}$ proposait la distinction suivante : le miraculum désignerait le miracle dont l'agent est Dieu, le mot mirabilia s'appliquerait au merveilleux en relation avec la nature, à ce qui étonne mais qui est naturel, et enfin magicum désignerait la magie dont l'efficacité est le plus souvent imputée au diable. Mais dans les textes eux-mêmes la définition de ces termes est fluctuante, avant tout parce que, dans l'esprit des hommes du Moyen Âge, il n'existait pas de catégorie mentale ou intellectuelle qui aurait permis de séparer le merveilleux du réel ou du quotidien.

S'il est difficile de délimiter la catégorie du merveilleux dans l'esprit médiéval, il est bien plus difficile encore de créer des subdivisions au cœur $\mathrm{du}$ merveilleux. On passe sans s'en rendre compte du domaine de l'intervention divine à des mirabilia plus neutres, dont l'agent n'est ni Dieu ni le diable, mais qui appartiennent quand même au domaine du surnaturel. Ainsi, la magie blanche est plutôt du côté des mirabilia, voire du miracle et Merlin fut considéré, entre le $\mathrm{XIII}^{\mathrm{e}}$ et le $\mathrm{XV}^{\mathrm{e}}$ siècle, comme un grand prophète par beaucoup de clercs, qui ont ainsi christianisé cette figure celtique de mage. Toutes les distinctions que l'on peut essayer de dresser sont plus des lignes de force ou des pôles de référence que des limites définitives.

La distinction la plus importante pour la compréhension de la logique médiévale pourrait être celle qui sépare le merveilleux reconnu - c'est-àdire reconnu par les autorités ecclésiastiques, donc voulu par Dieu et que l'on peut rattacher aux manifestations divines - du merveilleux non reconnu, et donc inquiétant, voire franchement suspect, parce que rattaché à la sphère diabolique.

\footnotetext{
1 Jacques Le GofF, «Le Merveilleux dans l'Occident médiéval », in L'Imaginaire médiéval : essais, Paris, Gallimard, 1985 (première publication sous la forme d'article en 1978).
} 


\section{Éléments pour une typologie}

Il semble difficile d'établir une typologie des manifestations divines tant leurs expressions sont variées et diverses. On peut toutefois mettre en avant certains phénomènes et certaines pratiques. La manifestation divine par excellence est la théophanie. Ce terme désigne l'apparition d'un dieu au cours de laquelle a normalement lieu la révélation aux hommes d'un message divin ou d'une mise en garde. Déjà utilisée dans la culture grecque, où elle désigne l'exposition des statues des dieux lors de fêtes, l'expression est reprise par le christianisme. Les principales théophanies de la Bible sont l'apparition du buisson ardent à Moïse dans l'Ancien Testament, et, dans le Nouveau Testament, la naissance de Jésus ou la Transfiguration. Les reliques achiropoiètes, c'est-à-dire qui n'ont pas été créées par la main de l'homme, sont à rapprocher de ces manifestations, car elles permettent un contact direct et sans médiation avec la divinité. Il s'agit principalement du saint suaire et du voile de Véronique, auquel le Moyen Âge facétieux et pragmatique ajoutait à l'occasion le cordon ombilical, le prépuce ou encore les dents de lait du Christ ${ }^{2}$. Faut-il compter au nombre des théophanies l'eucharistie où chaque dimanche, selon le dogme catholique, s'incarne la présence réelle du Christ? Elle est une preuve de plus de la quotidienneté du surnaturel.

L'actuel catéchisme catholique ne prend pas la peine de définir ce qu'est un miracle et la définition d'un dictionnaire est trop vaste pour avoir un intérêt scientifique. Peut-être peut-on reprendre celle que retient PierreAndré Sigal $^{3}$, tirée du Dictionnaire alphabétique et analogique de la langue française de Paul Robert: " un fait extraordinaire où l'on croit reconnaître l'action divine bienveillante, auquel on confère une

\footnotetext{
${ }^{2}$ Ainsi l'abbaye Saint-Médard de Soissons prétendait posséder une dent de lait du Christ.

${ }^{3}$ Pierre-André Sigal, L'Homme et le miracle dans la France médiévale (XI $-X I I^{e}$ siècle), Paris, Éditions du Cerf, 1985, p. 17.
} 
signification spirituelle $»^{4}$. Le miracle le plus commun est la guérison, miracle par excellence puisque c'est celui que le Christ pratique le plus volontiers dans la Bible. Mais on trouve aussi des miracles de punition ou encore des manifestations météorologiques qui acquièrent un statut miraculeux. Le point commun de toutes ces manifestations est que Dieu ne se manifeste pas directement mais qu'il utilise l'intercession du saint. L'homme médiéval considère que ce sont le saint et ses reliques qui guérissent, en accord avec la volonté divine. Le saint, grâce à ses rapports privilégiés avec Dieu, est le premier bénéficiaire des manifestations divines : visions, apparitions, voix, signes divins (apparitions d'animaux, stigmatisations). Il s'agit alors pour Dieu d'expliciter ce qu'il attend du saint et de le conforter dans sa vocation.

Dieu intervient également dans la grande histoire et les hommes n'hésitent pas à le solliciter lors de grands événements. À l'aube de batailles décisives, face à un ennemi impie, une prière fervente assure la victoire, à l'imitation de Constantin au Pont Milvius ${ }^{5}$. Lors d'un jugement, l'ordalie et le duel judiciaire reposent sur l'idée que Dieu ne peut laisser perpétrer une iniquité. Le combat devient alors le lieu d'expression de la volonté divine.

On pourrait ainsi proposer un classement des manifestations divines selon trois cercles concentriques de proximité avec Dieu. Le premier cercle contiendrait les apparitions et manifestations directes de Dieu comme l'eucharistie ou la manifestation de Dieu à un saint pour le guider. Le second cercle serait constitué des manifestations divines qui utilisent les

\footnotetext{
${ }^{4}$ Paul RoBert, Dictionnaire alphabétique et analogique de la langue française, Paris, 1959, t. IV, p. 590.

${ }^{5}$ Selon le récit d'Eusèbe de Césarée, repris par de nombreux auteurs tout au long du Moyen Âge, la conversion de Constantin au christianisme fait suite à l'apparition dans le ciel d'un chrisme à la veille de la victoire du Pont Milvius face à l'usurpateur Maxence. Suite à cette vision, Constantin fait peindre un chrisme sur les boucliers de ses hommes et se place sous la protection du Christ. Sa victoire est la preuve évidente du soutien de Dieu à ses ambitions impériales.
} 
saints pour vecteurs, que ce soit par le biais d'un miracle ou d'une relique. Le dernier cercle correspondrait aux manifestations moins évidentes, indirectes ou douteuses qui sont attribuées à Dieu sans qu'aucun indice de type vision ou apparition ne vienne le confirmer: une catastrophe météorologique ou une récolte étonnement abondante peuvent illustrer cette dernière catégorie. Bien sûr, ces trois cercles ne sont pas imperméables et beaucoup de récits pourraient être classés tantôt dans le deuxième tantôt dans le troisième cercle selon la façon dont les évènements sont présentés.

Comme nous le montre plus particulièrement le troisième cercle, la manifestation divine repose en grande part sur la lecture qu'en fait celui qui la rapporte et qui l'attribue à Dieu. Cette part d'interprétation confrontée à l'idéologie scientiste du $\mathrm{XIX}^{\mathrm{e}}$ siècle explique le long désintérêt des historiens pour ces phénomènes, que l'on ne peut encore aborder aujourd'hui sans se demander quel crédit leur accorder.

\section{Les manifestations divines dans l'historiographie}

Depuis le Romantisme, le Moyen Âge était perçu comme une époque «fantastique » où les forces surnaturelles liées à toutes sortes de croyances intervenaient directement dans la vie des humains. C'est l'idée que l'on retrouve chez Walter Scott, Victor Hugo ou Michelet: les hommes du Moyen Âge sont comme de grands enfants, ingénus, prêts à croire toutes les légendes et à en créer toujours de nouvelles.

La perspective apologétique du $\mathrm{XIX}^{\mathrm{e}}$ siècle, surtout chez les catholiques ultramontains, s'intéresse particulièrement à un âge de foi associé aux cathédrales et aux croisades par opposition au rationalisme sceptique des hommes de leur temps, qui prenaient la suite de Voltaire en tournant en dérision les phénomènes surnaturels en tant que superstitions et impostures. Le contexte est alors très polémique : l'histoire critique et 
positiviste se méfie des recueils de miracles et des collections de récits merveilleux. Les historiens en laissent l'étude aux spécialistes de la littérature et du folklore, car le travail de l'historien consiste à établir des « faits vrais » et ce genre de texte ne l'intéresse donc que pour fournir des anecdotes pittoresques, en marge de l'histoire. Les historiens se contentent de dégager les realia contenus dans les textes de l'enrobage merveilleux qui les recouvraient.

Le premier à s'intéresser à ces textes dans une perspective anthropologique est Marc Bloch, qui publie en 1924 Les Rois thaumaturges $^{6}$. Il s'efforce d'interpréter les récits merveilleux dans l'optique des catégories mentales des $\mathrm{XI}^{\mathrm{e}}$ et $\mathrm{XII}^{\mathrm{e}}$ siècles et ne se pose pas la question de savoir si les miracles sont vrais ou faux. Il s'interroge plutôt sur ce qu'ils révèlent de ceux qui y participent. La croyance aux miracles devient une donnée constitutive de la mentalité médiévale et il est donc logique d'en étudier les implications dans le domaine religieux et politique.

C'est une œuvre pionnière mais qui connaît peu de suite jusqu'aux années 1960 où la vogue de l'histoire des mentalités s'accompagne d'un nouvel intérêt pour la religion populaire et la culture folklorique. Le miracle et le merveilleux deviennent alors des objets d'études à part entière. En 1978, Jacques Le Goff publie un article intitulé «Le Merveilleux dans l'Occident médiéval ${ }^{7}$ et fait ainsi entrer le merveilleux dans le domaine de l'historien, par réaction face aux interprétations trop exclusivement littéraires ou esthétisantes. Le Moyen Âge pour Jacques Le Goff ne mérite nullement l'épithète de « fantastique ». Ces réflexions sont

\footnotetext{
${ }^{6}$ Marc Bloch, Les Rois thaumaturges : étude sur le caractère surnaturel attribué à la puissance royale particulièrement en France et en Angleterre, Paris-StrasbourgLondres, Istra-Humphrey Milford-Oxford University Press, 1924.

${ }^{7}$ Art. cit.
} 
prolongées par Daniel Poirion ${ }^{8}$ et Claude Lecouteux ${ }^{9}$ dans le domaine littéraire, par Claire Kappler ${ }^{10}$ sur la question des monstres, par JeanClaude Schmitt sur la question des revenants ${ }^{11}$, et aboutissent à la synthèse de Michel Meslin ${ }^{12}$ qui élargit le cadre de sa réflexion à l'époque moderne et contemporaine.

Parallèlement l'histoire connaît un renouveau d'intérêt pour les miracles avec Pierre-André Sigal ${ }^{13}$, André Vauchez ${ }^{14}$ ou Benedicta Ward ${ }^{15}$, et la multiplication des travaux sur l'hagiographie depuis les années 1980. On assiste donc à une évolution tardive mais très rapide qui fait du merveilleux, et en particulier du merveilleux chrétien, un domaine à part entière dans le travail de l'historien. Cette meilleure connaissance du phénomène nous permet de ne plus envisager le merveilleux chrétien au Moyen Âge comme un bloc homogène mais de découvrir les subtilités de mille ans d'évolution.

\footnotetext{
${ }^{8}$ Daniel PoIRIOn, Le Merveilleux dans la littérature française du Moyen Âge, Paris, Presses Universitaires de France, "Que sais-je ? », 1982.

${ }^{9}$ Claude Lecouteux, Fantômes et revenants au Moyen Âge, Paris, Imago, 1986, Fées, sorcières et Loups-garous : histoire du double au Moyen Age, Paris, Imago, 1992, Audelà du merveilleux: des croyances au Moyen Âge, Paris, PUPS, 1995 et Chasses fantastiques et cohortes de la nuit au Moyen Âge, Paris, Imago, 1999, pour ne citer que quelques titres.

${ }^{10}$ Claire KAPPler, Monstres, démons et merveilles à la fin du Moyen Âge, Paris, Payot, 1988.

${ }^{11}$ Jean-Claude SchmiTt, Les Revenants. Les vivants et les morts dans la société médiévale, Paris, Gallimard, 1994.

${ }^{12}$ Michel Meslin (dir.), Le Merveilleux : l'imaginaire et les croyances en Occident, Paris, Bordas, 1984.

${ }_{13}^{13}$ Pierre-André Sigal, L'Homme et le miracle dans la France médiévale, op. cit.

14 André Vauchez, La Sainteté en Occident aux derniers siècles du Moyen Âge, d'après les procès de canonisation et les documents hagiographiques, Rome, EFR, 1981.

15 Benedicta WARD, Miracles and the Medieval Mind: Theory, Record and Event (1000-1215), Philadelphia, University of Pennsylvania Press / London, Scolar press, 1982.
} 


\section{Évolution des manifestations divines au Moyen Âge}

$\mathrm{Au}$ Haut Moyen Âge, tout ce qui est insolite est mirum ou stupendum et le surnaturalisme est sans nuance. Progressivement les pratiques païennes sont christianisées, les dons des héros ou des druides sont attribués aux saints, les fêtes sont reprises et adaptées aux besoins de l'Église. Ainsi la fête de la Chandeleur s'inscrit-elle dans le calendrier à la fois à la place de la fête romaine des Lupercales et d'une fête celtique, Imbolc, fête dédiée à Brigit pour assurer la purification et la fécondité ; la date est désormais associée à la purification de la Vierge et à la présentation du Christ au Temple, mais devient aussi une fête de la lumière dans un cadre chrétien.

À partir de saint Anselme et de Guibert de Nogent, au XI ${ }^{\mathrm{e}}$ siècle, l'attitude des élites cultivées change et ces dernières prennent progressivement leurs distances par rapport à la piété populaire. Les phénomènes surnaturels sont de plus en plus placés sous le contrôle des institutions qui leur donnent un sens. Les manifestations divines atteignent leur apogée aux XII ${ }^{\mathrm{e}}$ et XIII ${ }^{\mathrm{e}}$ siècles. Saint Bernard de Clairvaux (10901153) fut le dernier grand faiseur de miracles in vita de la chrétienté occidentale. En revanche saint François d'Assise ( 1180-1226) est beaucoup plus sobre dans les actions qu'il accomplit, quoiqu'il reçoive les stigmates. Mais c'est là une nouvelle forme de manifestation divine, et non plus l'action de Dieu par l'intermédiaire du saint comme dans le cas classique du miracle. Le miracle ne se produit plus in vita mais post mortem et devient condition de sainteté retenue pour les procès en canonisation. Entre la deuxième moitié du $\mathrm{XII}^{\mathrm{e}}$ siècle et le début du XIII ${ }^{\mathrm{e}}$ siècle, beaucoup de traités intitulés De miraculis ou Libri miraculorum sont rédigés, par exemple par Pierre le Vénérable ( 1092-1156), Césaire d'Heisterbach ( 1180-avant 1250) ou Thomas de Celano ( 1190- 1260). 
Le miraculum sert de plus en plus à défendre la foi chrétienne et rejoint le rôle des exempla dans la prédication.

$\mathrm{Au} \mathrm{XIII}^{\mathrm{e}}$ siècle, on voit apparaître les premières critiques rationalistes qui entraînent un rétrécissement du champ des mirabilia. Gervais de Tilbury est le premier à établir une distinction nette entre les miracles et les merveilles ${ }^{16}$ : les premiers renvoient à la toute-puissance divine alors que les secondes obéiraient aux lois naturelles et n'apparaîtraient comme extraordinaires qu'à cause de notre ignorance, ce qui suppose qu'un jour on pourrait les expliquer. Le miraculeux se restreint à certains prodiges riches en enseignements et pouvant être attribués à la puissance divine. Au XIII ${ }^{\mathrm{e}}$ siècle également, les clercs œuvrent pour un endiguement du merveilleux en cherchant à le rattacher à des objets plus dignes de foi que des reliques souvent douteuses. Ils orientent la piété des fidèles vers la dévotion à la Vierge et les sacrements de l'autel. Ces nouvelles dévotions sont soutenues par la littérature religieuse, surtout en langue vernaculaire, et entraînent le développement du merveilleux marial et du merveilleux autour du saint sang. À partir de la fin du XII ${ }^{\mathrm{e}}$ siècle, mais s'amplifiant beaucoup par la suite, un phénomène de désenchantement du monde touche d'abord les élites et les clercs. La scolastique et Thomas d'Aquin jouent un rôle dans cette évolution: le miracle ne peut être qu'un phénomène supra naturam, c'est-à-dire au dessus des lois de la nature, car il est exclu que Dieu agisse contra naturam, c'est-à-dire contre les lois de la nature qu'il a lui-même édictées. Ce raisonnement donne une délimitation plus stricte à la sphère du merveilleux.

La fin du XIII ${ }^{\mathrm{e}}$ siècle est le moment d'une nouvelle donne pour le merveilleux chrétien. Les théologiens mettent en évidence des modalités

${ }^{16}$ Gervais de Tilbury, Le Livre des Merveilles. Divertissement pour un Empereur, (troisième partie), traduction et commentaires par Annie DUCHESNE, Paris, Les Belles Lettres, 1992. 
inédites de coopérations entre la nature et la grâce qui se traduisent par la capacité qu'a la puissance divine de passer dans le corps pour agir sur la nature, par exemple dans le cas de la stigmatisation, dont les occurrences se multiplient après saint François d'Assise. Ce n'est donc pas vraiment un repli du surnaturel mais bien plutôt une mutation du merveilleux. À partir $\mathrm{du}$ début $\mathrm{du} \mathrm{XIV}^{\mathrm{e}}$ siècle, on assiste au développement de phénomènes mystiques tels que visions et révélations qui entraînent l'élaboration par les théologiens de traités sur le discernement des esprits, œuvres où il s'agit d'établir si ces phénomènes sont d'origine divine ou diabolique. L'augmentation de la fréquence des apparitions renforce la position du diable car la place reconnue à l'expérience intérieure et à la subjectivité augmente l'ambiguïté de ces phénomènes. Les manifestations les plus grossières de la croyance au merveilleux, magie puis sorcellerie, sont de plus en plus combattues. On a aussi de plus en plus souvent recours aux interprétations apocalyptiques des évènements historiques : les phénomènes météorologiques ou astrologiques inexpliqués offrent des lectures plurielles qui penchent soit pour le signe d'un dérèglement de l'Église ou de la société, soit pour un présage de l'âge d'or à venir.

\section{Adaptations littéraires}

On retrouvera dans le domaine de la littérature les problématiques généralement associées aux manifestations divines et liées aux questions de représentation et d'interprétation.

\section{Le poids du modèle biblique}

Pour l'auteur médiéval désireux de décrire une intervention divine, la Bible constitue à la fois un répertoire de situations et un modèle d'écriture incontournable. Cependant, la Bible présente elle-même plusieurs manières de faire intervenir Dieu. 
Dans l'Ancien Testament, il existe certes des phénomènes surnaturels manifestant la volonté divine, mais ce sont des phénomènes affectant la nature - tout phénomène inexplicable (ouverture de la Mer Rouge, plaies d'Égypte, buisson ardent...), aussi appelé prodige, doit être interprété comme message de Dieu. L'Ancien Testament montre en outre un large éventail d'interventions angéliques auprès des Élus: auprès d'Abraham, de Jacob, de Loth, etc. Quant à Dieu lui-même, il se manifeste de préférence sous l'aspect d'une voix, comme au mont Tabor.

Le Nouveau Testament développe de nouvelles manières de faire intervenir le divin. L'on peut y constater une certaine continuité avec l'Ancien Testament; on y trouvera de nouveau des messages angéliques (annonce aux bergers, Annonciation...), voire la voix de Dieu intervenant lors du baptême du Christ. Mais aux prodiges et aux messages s'ajoutent désormais les miracles, des faits extraordinaires que le témoin attribuera d'emblée à une action divine bienveillante, et auxquels il confèrera une signification spirituelle. Si l'auteur du miracle peut être Dieu, le Christ, mais aussi - par délégation - un ange ou un saint, les premiers miracles sont toujours centrés sur la personne du Christ : l'intervention de Dieu lors de son baptême, son don de thaumaturge, sa capacité à transformer l'eau en vin ou à marcher sur les eaux sont destinés à manifester de manière spectaculaire sa nature divine aux non-croyants. Le Christ peut néanmoins transmettre ses pouvoirs - sa vertu ${ }^{17}$ - à ses apôtres, voire à quiconque croit en lui. La foi est ainsi le pré-requis du miracle, mais aussi son objet : un miracle sert essentiellement à permettre la propagation rapide de la foi auprès des non-croyants. En effet, il n'a pas à être expliqué et ne fait pas objet de questionnement : il est monstrance et demonstrance immédiate de la toute puissance de Dieu par-delà les lois de la nature.

17 Vertu est souvent en ancien français le terme désignant le pouvoir surnaturel de l'auteur de miracles. 
La littérature religieuse, de l'hagiographie au mystère: le problème de la représentation

Au Haut Moyen Âge demeure encore, chez les Pères de l'Eglise, une certaine répugnance à laisser se développer la reconnaissance des miracles accomplis par les saints, mais cette réticence cède peu à peu du terrain et le culte des saints, des reliques et les pèlerinages qui leur sont associés peuvent alors prendre leur plein essor. Parallèlement sont rédigées de nombreuses hagiographies mettant en évidence les pouvoirs surnaturels que la foi confère aux saints ; ces pouvoirs sont alors considérés comme les signes de l'approbation divine dont ils bénéficient. Les théophanies au sens strict y sont rares, car elles supposent une immense grâce accordée au spectateur. De plus, représenter la divinité est sans doute un défi que tout auteur n'est pas prêt à relever. En revanche, les hagiographies mettent en scène de nombreuses interventions angéliques, voire des interventions de la Vierge, et présentent fréquemment les saints eux-mêmes comme étant à l'origine de ces faits surnaturels où se manifeste le sacré.

Nous détaillerons ici les divers éléments qui composent dans les textes ce "miracle de la sainteté ${ }^{18}$. Tout d'abord la destruction du corps du saint montre que la gloire religieuse doit paradoxalement s'associer à une humiliation mondaine. Le corps martyrisé est alors exposé avec un certain sadisme qui rappelle la nécessité de détruire tout ce qui est lié au paganisme, dont, notamment, le corps et la sexualité. Le saint a en outre le privilège de la communication avec Dieu ; il peut recevoir ou provoquer un message divin. En faveur d'Alexis, dans l'hagiographie éponyme ${ }^{19}$, c'est une image de la Vierge, faite par les anges, qui un jour se met à parler : la volonté divine se manifeste ainsi de manière indirecte, tout en conservant

\footnotetext{
${ }^{18}$ Nous empruntons cette analyse à Daniel POIRION, Le Merveilleux dans la littérature française du Moyen Âge, op. cit.

${ }^{19}$ La Vie de saint Alexis, édition critique par Maurizio Perugi, Genève, Droz, « Textes littéraires français », $\mathrm{n}^{\circ} 529,2000$.
} 
un des traits de l'oracle antique, car il faudra en déchiffrer l'énigme. Être en communication directe avec Dieu est donc le privilège de l'Élu : c'est pourquoi, n'osant se proclamer tel, l'écrivain médiéval (aux XII ${ }^{\mathrm{e}}$ et $\mathrm{XIII}^{\mathrm{e}}$ siècles du moins) ne revendique ni magie ni enthousiasme à l'origine de son œuvre ; par modestie, il rejette le modèle du furor emprunté aux latins. Mais il suggère la transmission d'une signification divine par l'Écriture. Le saint peut aussi relayer la toute-puissance divine. Il est aidé par la Providence, qui peut lui accorder le pouvoir divin, mais cette dernière le met néanmoins à l'épreuve. Ainsi Marguerite échappe-t-elle à la mort par le feu et par l'eau grâce au Saint Esprit : le sacré se sur-imprime alors sur le magique en le chargeant de sens. Paradoxalement, la présence de forces diaboliques venues le combattre ou le tenter sont d'autres signes sûrs de la sainteté d'un personnage. Dans les hagiographies, les créatures monstrueuses issues d'autres cultures sont constamment présentées comme diaboliques. Les diables peuvent ainsi ressembler à des satyres ou des Priape de l'Antiquité, ou peuvent même prendre la forme de dragons ${ }^{20}$. Enfin, l'horizon de l'existence du saint est celui du sacrifice d'Abraham et de la Passion du Christ; cela implique a minima un renoncement à la sexualité par désir de purification, mais plus radicalement la capacité à renoncer à la vie par obéissance ou par charité.

Dans les représentations théâtrales du sacré, les mystères, la manifestation divine pose en outre le problème de la mimesis, qui bute sur la représentation du surnaturel. Chaque auteur résout la difficulté avec plus ou moins de bonheur : au début du XIII ${ }^{\mathrm{e}}$ siècle, Le jeu de Saint Nicolas, de Jean Bodel $^{21}$, met en scène le merveilleux épique de la croisade en le

\footnotetext{
${ }^{20}$ Ainsi, dans le Merlin de RoBerT DE BORON, où il est dit que la mère de Merlin a été fécondée par un incube ; ou dans La Vie de sainte Marguerite, où la sainte affronte un dragon qui se révèle n'être autre que le Diable.

${ }^{21}$ JeAn Bodel, Le Jeu de saint Nicolas, Jean Dufournet (éd.), Paris, Flammarion, 2005.
} 
mêlant au merveilleux hagiographique d'un miracle. Mais le merveilleux est victime de la fonction même de la représentation, qui est de faire voir : comment représenter le surnaturel ? L'intervention divine se réduit donc à un discours de l'ange affirmant que les chevaliers morts sont recueillis par Dieu. Dans les Miracles de Notre Dame de Gautier de Coincy ${ }^{22}$, l'intervention de la Vierge devient une utilité du type deus ex machina chargée de résoudre les tensions et de mettre fin au récit. Dans le Miracle de Théophile $^{23}$, Rutebeuf crée une antithèse entre la magie noire de Saladin conjurant le diable et le miracle de la Vierge : l'auteur parvient cette fois-ci à intégrer l'intervention divine à son récit en créant un exemplum sur les enjeux d'un « écrit de sang », c'est-à-dire d'un pacte avec le Mal.

Les interventions divines dans la littérature narrative profane: miracle ou merveilleux?

Certaines caractéristiques du récit hagiographique se retrouvent à l'occasion dans les chansons de geste : dans la Chanson de Roland, saint Gabriel envoie des songes prémonitoires à Charlemagne (il l'enverra aussi en mission dans la dernière laisse de la chanson) et vient recueillir le gant offert par Roland à l'agonie. En lui accordant certains des privilèges du saint - communication avec Dieu et interventions angéliques - le jongleur cherche manifestement à assimiler le héros épique à un saint combattant pour la foi, à un martyr dont la cause est celle de la chrétienté24.

\footnotetext{
${ }^{22}$ Gautier De CoInCy, Cinq miracles de Notre Dame, traduction de Jean-Louis Gabriel BENOIT, Paris, Honoré Champion, «Traduction des classiques français du Moyen Âge », 2007.

${ }^{23}$ Rutebeuf, Le Miracle de Théophile, Jean Dufournet (éd.), Paris, Flammarion, 1987.

${ }^{24}$ La Chanson de Roland, Cesare SEgRe (éd.), Genève, Droz, «Textes littéraires français ", 2003, laisse CLXXVI, p. 212. La chanson fait aussi assister au miracle du soleil arrêté par Dieu sur la prière de Charlemagne (laisses CLXXIX-CLXXX, v. 24432475 , p. 215-217.) On peut néanmoins remarquer que le merveilleux chrétien, à l'exception notable de ces quelques scènes, se fait discret dans cette chanson : la faveur divine ne doit pas diminuer le mérite des héros.
} 
Cependant, dans la littérature romanesque, la logique du miracle rencontre le plus souvent une autre logique, cette fois-ci profane, qui est celle de la merveille. Cette dernière se définit par la réaction qu'elle provoque, un étonnement nuancé de crainte, d'admiration ou de fascination. Souvent tirée d'un autre système culturel, elle renvoie à un passé ou un ailleurs, et notamment à des mythes archaïques issus de la mythologie antique ou celtique. Elle peut aussi naître d'un autre type de décalage, situé entre la culture savante et la culture populaire. Le merveilleux manifeste ainsi un écart culturel entre des valeurs de référence, qui servent à établir le socle de communication entre l'auteur et son public, et les qualités d'un monde autre.

Le miracle appartient au vaste domaine de la merveille, mais il en réduit l'extension sémantique en caractérisant un seul et même événement surnaturel comme relevant de l'intervention providentielle. La mentalité médiévale ne perçoit originellement pas de délimitation stricte entre merveilleux chrétien et merveilleux païen. Il coexiste des manifestations magiques relevant ou non de pouvoirs surnaturels dont sont dotés des mortels, et des manifestations relevant du divin, de l'intervention de la Providence dans l'Histoire. Il en va ainsi dans les plus anciens textes de fiction que nous possédons. Nous prendrons l'exemple du Voyage de saint Brendan, odyssée chrétienne inspirée des imrama celtiques. C'est le récit d'un voyage initiatique du saint vers l'Autre Monde. Dans ce texte latin du $\mathrm{IX}^{\mathrm{e}}$ siècle, le héros croise une succession de lieux fantastiques, inspirée par la Navigation de Bran et d'autres récits celtiques, mais aussi par l'Énéide, l'Odyssée, ou les voyages de Sinbad dans les Mille et une nuits. Merveilleux profane et merveilleux sacré se trouvent ainsi inextricablement mêlés.

Le glissement de la perception d'un événement surnaturel merveilleux à une interprétation mettant en jeu une intervention divine 
nécessite un acte de foi identifiant l'action de la Providence. La chance extraordinaire de Tristan qui, lorsqu'il saute de la chapelle au bord de la falaise, atterrit au pied de cette falaise sans se faire mal, pourrait n'être qu'un signe du caractère surhumain du héros. Pourtant Béroul choisit une autre interprétation en notant que «Bele merci Dex li a fait $! »^{25}$. Francis Dubost remarque ainsi qu'un même événement peut se voir qualifié successivement de merveille et de miracle ${ }^{26}$. L'identification du miracle peut du reste être sujette à erreur : ainsi, dans Le Conte du Graal, Perceval croit voir des anges lorsqu'il rencontre les chevaliers pour la première fois. Tout événement inexplicable ne doit pas être imputé d'office à une intervention divine ; identifier celle-ci relève d'un choix interprétatif qui n'est pas infaillible.

Peu à peu, l'Église s'efforcera de faire disparaître des récits toute manifestation merveilleuse de type profane: les fées deviendront des prophétesses, Merlin, autrefois figure druidique, un prophète inspiré par Dieu à l'origine de la quête du Graal, comme chez Robert de Boron ${ }^{27}$. À l'inverse, d'autres manifestations de pouvoir de type magique seront rejetées du côté de la magie noire, du démoniaque. Le mouvement perceptible est celui d'un effort de rationalisation constante, une tentative d'intégrer le merveilleux pré-chrétien à la vision chrétienne du monde.

25 Beroul, Tristan, dans Les Tristan en vers, Jean-Charles PAYen (éd.), Paris, Bibliopolis, 1999 [texte numérisé reproduisant l'édition de Paris, Bordas, 1991]. Le récit du saut de la chapelle est relaté aux vers 917-935.

${ }^{26}$ Francis DuBOST, Aspects fantastiques de la littérature narrative médiévale : XII ${ }^{e}$-XIII siècles: l'autre, l'ailleurs, l'autrefois, Paris, Honoré Champion, « Nouvelle bibliothèque du Moyen Âge » 15, 2010 ( $1^{\text {ère }}$ édition en 1991), p. 85.

${ }^{27}$ Robert De Boron, Merlin, Alexandre Micha (éd.), Paris, Flammarion, GF, 1994. Dans ce récit, l'auteur modifie en profondeur l'image de Merlin héritée de l'œuvre de Geoffroy de Monmouth (Vie de Merlin et Histoire des rois de Bretagne) : Merlin y devient le pivot d'un affrontement de type eschatologique entre le Christ et le Diable. Fils d'un démon, il est sauvé par son baptême et la foi de sa mère et devient alors un fidèle serviteur du Christ. 
La dichotomie entre merveilleux chrétien et miracle subsiste néanmoins. Le miracle est univoque, alors que le merveilleux chrétien épaissit le mystère. Nous citerons à ce sujet cette réflexion de Francis Dubost :

(...) miracle et merveille se partagent l'espace littéraire. Le miracle est en position dominante dans la chanson de geste ; alors que, dans le roman, et spécialement le roman arthurien, c'est la merveille qui l'emporte. Le miracle est conclusif et résolutif; la merveille, très souvent inassignable, au moins dans un premier temps, ouvre une crise, instaure une épreuve, dresse un obstacle et sollicite les ressources humaines et surhumaines. ${ }^{28}$

Si le miracle comble avec la spontanéité de l'évidence, la merveille en revanche crée une interrogation, une épreuve ${ }^{29}$.

Le merveilleux, qu'il soit issu du paganisme ou qu'il ait été christianisé, comme dans le cas des manifestations divines, a longtemps été considéré comme la trace et l'expression d'une mentalité archaïque, imprégnée d'irrationnel et de surnaturel. On sait aujourd'hui qu'il est impossible de séparer vérité et merveilleux, car les discours médiévaux sont des constructions homogènes et ordonnées où thèmes merveilleux et données réalistes s'articulent pour produire un ensemble à la symbolique cohérente.

Les manifestations divines, dans leur diversité et leur omniprésence, invitent le chercheur contemporain à dépasser ses préjugés cartésiens et à s'interroger à la fois sur les rapports entre les hommes et Dieu et sur les utilisations possibles de ces manifestations par l'humanité. Dans le premier cas, les récits des manifestations divines sont l'occasion d'exprimer le

\footnotetext{
${ }^{28}$ Francis Dubost, Aspects fantastiques de la littérature narrative médiévale, op. cit., p. 85 sqq.

${ }^{29} \mathrm{Au}$ sens où elle donne matière à réflexion sans fournir de réponse univoque : il en va ainsi dans les cortèges du Graal, les songes prophétiques, les inscriptions prophétiques sur des tombes, etc.
} 
sublime, de dire l'indicible, ce qui représente souvent un défi pour l'auteur. Dans le second cas, il s'agit de s'interroger sur ce qui motive la mise par écrit de tels évènements. Si le récit d'une manifestation divine n'a pas pour but de berner celui qui en est le spectateur ou le lecteur et qu'il se propose de répondre à la curiosité des hommes en expliquant l'inexplicable, il occupe aussi une place importante dans les phénomènes de compensation, pour limiter les tensions sociales et faire prévaloir la protection de l'humble. Il n'est pas non plus dénué d'intention politique et sert à la légitimation des pouvoirs tant laïcs qu'ecclésiastiques. Ces deux axes de réflexion constituent, pour ainsi dire, les deux faces d'une même pièce que nous retrouvons dans les articles qui suivent. 